\title{
ALFA: First Operational Experience of the MPE/MPIA Laser Guide Star System for Adaptive Optics
}

\author{
R. I. Davies ${ }^{a}$, W. Hackenberg ${ }^{a}$, T. Ott ${ }^{a}$, A. Eckart ${ }^{a}$, H.-C. Holstenberg ${ }^{a}$, \\ S. Rabien ${ }^{a}$, A. Quirrenbach ${ }^{b}$ and M. Kasper ${ }^{c}$ \\ ${ }^{a}$ Max-Planck-Institut für extraterrestrische Physik, 85740 Garching, Germany \\ ${ }^{b}$ University of California, San Diego \\ ${ }^{c}$ Max-Planck-Institut für Astronomie, Königstuhl 17, 69117 Heidelberg, Germany \\ See paper 3353-05 for other MPIA project members and report on the AO system
}

\begin{abstract}
The sodium laser guide star adaptive optics system ALFA has been constructed at the Calar Alto 3.5-m telescope. Following the first detection of the laser beacon on the wavefront sensor in 1997 the system is now being optimized for best performance. In this contribution we discuss the current status of the launch beam and the planned improvements and upgrades. We report on the performance level achieved when it is used with the adaptive optics system, and relate various aspects of our experience during operation of the system. We have begun to produce scientific results and mention two of these.
\end{abstract}

Keywords: adaptive optics, laser guide star, launch beam, tip-tilt, sodium layer, atmospheric turbulence

\section{INTRODUCTION}

ALFA is a laser guide star adaptive optics system for the 3.5-m telescope at the German-Spanish Centre for Astronomy on Calar Alto, with a performance goal of achieving $50 \%$ Strehl at $2.2 \mu \mathrm{m}$ under typical seeing conditions $\left(0.9^{\prime \prime}\right)$ with good sky coverage. It has been built as a joint venture between the Max-Planck-Institut für Astronomie (Heidelberg) responsible for the wavefront sensing and adaptive optics, and the Max-Planck-Institut für extraterrestrische Physik (Garching) who provide the laser guide star.

The AO system utilises a Shack-Hartmann sensor with several lenslet arrays which can be interchanged, normally providing 6 or 18 subapertures (arranged in a hexagonal pattern) which are sampled at rates of $60-900 \mathrm{~Hz}$. The centroids of the spots in the sensor are used to determine the coefficients for Zernike or Karhunen-Loeve modes, and these are then applied to a 97-actuator mirror. When correcting on a natural guide star, the wavefront sensor determines all the modes including tip and tilt. When using the laser guide star a separate tracker is used to measure tip and tilt, since the up-link and down-link tip-tilt motions associated with alaser beacon are correlated. This part of the project is described in more detail in this proceedings and elsewhere. 1 .

The optical bench for the laser is installed in the coudé lab, where an $\mathrm{Ar}^{+}$laser with a $25 \mathrm{~W}$ multiline output pumps a dye ring laser with a single line output power of $4.25 \mathrm{~W}$. The lasers are continuous-wave to azpid difficulties of synchronisation with the AO. A detailed description of them can be found in other proceedings. beam is fed along the coudé path and directed into a launch telescope. At zenith the resulting sodium beacon has a magnitude of about $\mathrm{m}_{\mathrm{V}}=9-10$. Progress on optimizing the entire system has been hampered by poor weather, but important milestones include closing the loop on the laser beacon in Sept 1997, and then using it to enhance image resolution in Dec 1997 when 6 subapertures were sampled at a rate of $60 \mathrm{~Hz}$, allowing correction of 7 modes (plus tip and tilt) with a disturbance rejection bandwidth of approximately $6 \mathrm{~Hz}$. The latest result from March 1998, is that we have seen the laser guide star on the wavefront sensor using 18 subapertures at a frame rate of $100 \mathrm{~Hz}$ (Figure 5 ). We did not try to lock on it due to some focussing difficulties, although the signal-to-noise ratio suggests that this should be possible.

Other information:

E-mail: davies@mpa-garching.mpg.de

ALFA homepage: http://www.mpia-hd.mpg.de/MPIA/Projects/ALFA/index.html 
In this contribution we report on the current status of the laser system, and discuss issues that have arisen from our operational experience. We describe proposed experiments and plans for the near future. We have begun to produce scientific results, not only with the adaptive optics, but using the laser beacon as a reference and we include a section relating these.

\section{THE LAUNCH BEAM}

The design of the laser system incorporates an optical bench in the coudé lab on which the Coherent INNOVA 200 Pump and Coherent 899 dye lasers are installed, as there are facilities for providing the $5 \mathrm{~kW}$ pump power, cooling water, and dye circulation system. Preliminary control features are included at this stage. 3 Figure 1 shows how the output beam is directed by a succession of mirrors along the coudé path to the telescope where it is picked off near the primary mirror and directed into the Cassegrain launch telescope. There is another optical bench here where further diagnostic instruments can be installed before the beam is projected upwards.

\subsection{Current Status}

There are a number of components on the optical bench which are designed to optimize the quality of the launch beam, and hence the size and power of the laser beacon. These are described in the order in which the output beam from the dye laser encounters them.

\subsubsection{Line-width}

A small fraction of the beam power is diverted to a reference cavity which, for absolute wavelength tuning and long-term stabilisation, is locked to the Lamb dip in the fluorescence signal from a sodium cell. In principle this allows the laser line-width to be reduced to around $1 \mathrm{MHz}$ but in practice it is sufficient to keep it around $10 \mathrm{MHz}$, the natural line-width of the transition. It also allows the laser to be de-tuned away from the Na line for diagnostic purposes.

\subsubsection{Polarisation}

The ${ }^{2} \mathrm{~S}_{1 / 2}$ ground state of the $\mathrm{Na}$ atom is split into a doublet with a separation much greater than the laser line-width, and the laser is tuned to excite atoms from the higher angular momentum level $(F=2)$. Since they can decay back to either the $F=1$ or $F=2$ levels, after a few cycles the whole population will reside in the $F=1$ level, resulting in a significant loss of pump efficiency even though the atoms are replenished by high altitude winds. On the other hand, if the laser beam is circularly polarised so that it imparts angular momentum to the atoms, they will tend to decay back to the $F=2$ level, increasing the efficiency.

Since the output from the dye laser is plane-polarised, it can be circularly polarised simply by including a $\lambda / 4$ plate in the path. We expect a gain of 10-20\%, but with two stipulations. The first is that between the coudé lab and the launch telescope there are many reflections so that the final polarisation state of the beam is uncertain, probably elliptical. The second is that the polarisation only becomes an important issue if the beam is close to saturating the Na layer, otherwise the loss of atoms from the $F=2$ state is a minor effect.

Preliminary experiments suggest that currently the beam polarisation does not significantly affect the beacon brightness, and that the low power and large spot size are the major limitations.

\subsubsection{Beacon size}

The angular size of the laser beacon needs to be minimised so that it is more intense and the centroid better defined. Additional errors can arise with a LGS larger than the isoplanatic patch at the wavelength of the science observations, particularly if the size of the apertures on the wavefront sensor is well matched to the coherence length $r_{0}$. However, this is not a serious problem for us, since our system is designed to operate in the near-infrared where $r_{0}$ is relatively large.

The projected beam is focussed on the Na layer from a Cassegrain launch telescope. There is a pre-expander on the optical bench which increases the beam width to $1.5-2.5 \mathrm{~cm}$ diameter, controlling the size of the beam exiting the launch telescope within the range $29-49 \mathrm{~cm}$. Two conflicting effects determine the diameter used: the theoretical minimum spot size is reduced if a wider exit-beam diameter is used due to diffraction effects; but once this exceeds a few times the coherence length $r_{0}$, atmospheric turbulence will act to increase the spot size. In order to improve 


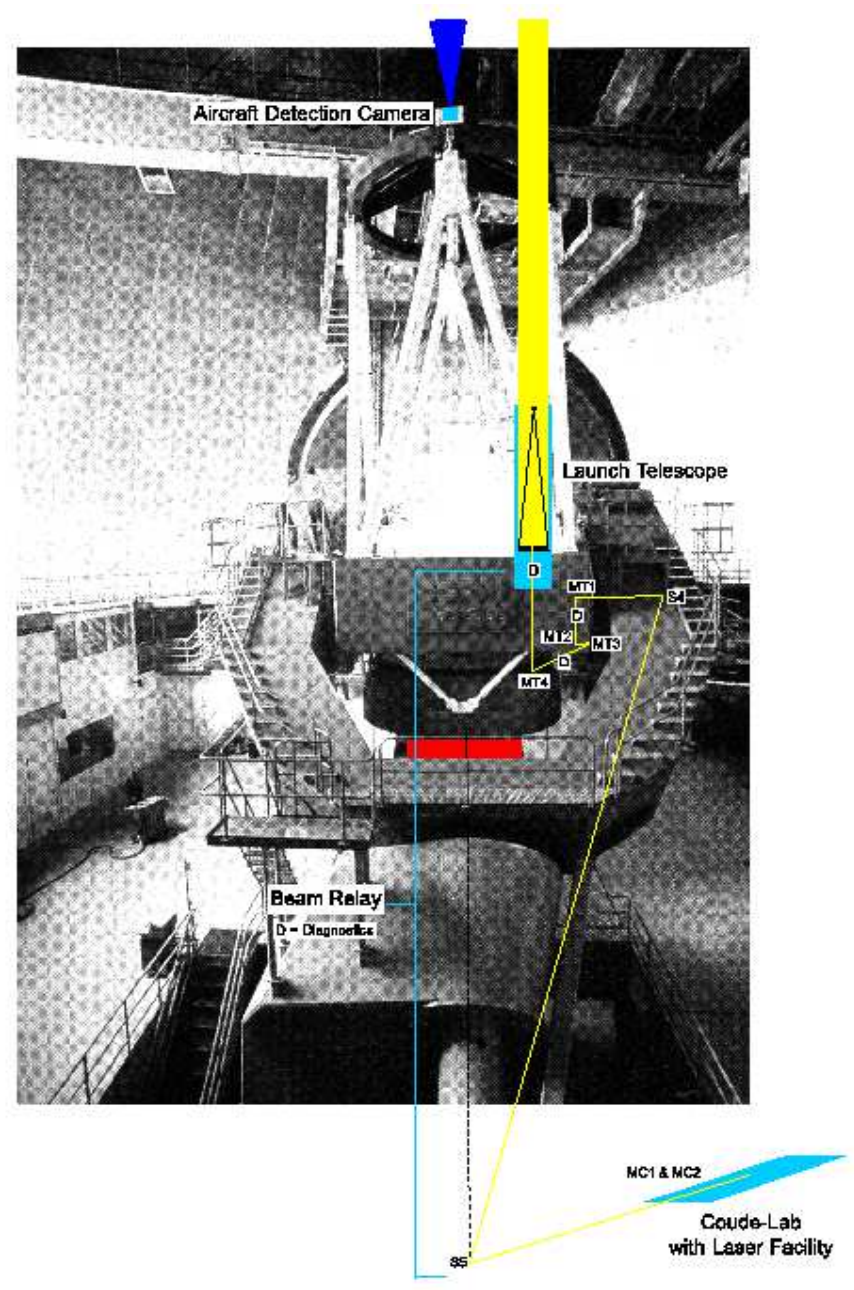

Figure 1. The 3.5-m telescope at Calar Alto and the ALFA LGS-system. The beam relay from the coudé lab is shown, with the position of the launch telescope and the aircraft detection camera ALIENS. Alignment of all the mirrors is achieved by use of diagnostic cameras (denoted D) installed at critical points.

on this, the laser is optimized to operate in a single (Gaussian) mode so that most of the power is concentrated in a small region.

Typically we achieve a spot size of $3^{\prime \prime}$, most of which is probably a result of dome seeing and turbulence arising between the coudé lab and telescope, for example due to temperature differences between the coudé lab and dome. To combat this we have already enclosed the entire path in a pipe, a scheme that produced a noticeable improvment and also acts as a safety precaution.

\subsubsection{Brightness}

The routinely obtainable laser output power is $4.25 \mathrm{~W}$. However, this is significantly reduced to about $60 \%$ by the time it reaches the telescope due to the multiple reflections from mirrors which very quickly become dirtied with dust blown over from the Sahara. 


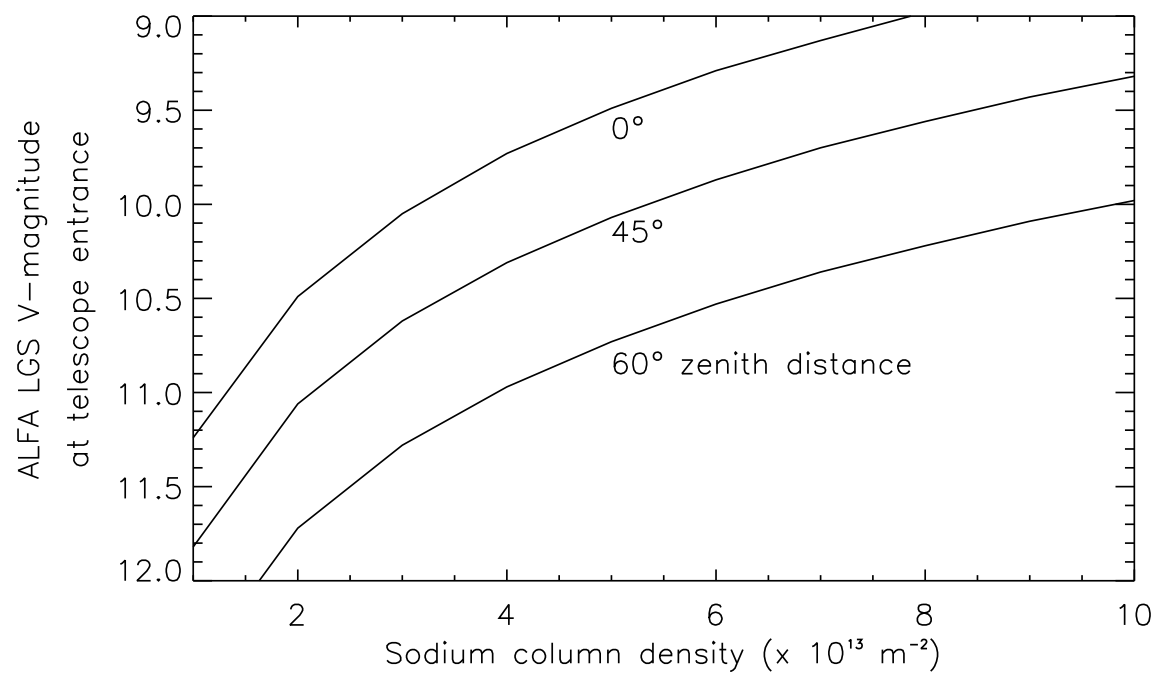

Figure 2. Predicted LGS brightness (given as equivalent V-band magnitude) as a function of zenith angle and $\mathrm{Na}$ column density, assuming a total transmission for excitation (including atmosphere) of $50 \%$.

The addition of COT to the Rh6G glycol dye solution increases the laser power by about $10 \%$ by further suppressing the triplet states in the dye molecules. However, we have found that this lasts only for short time-scales of tens of minutes. A further effect is that the lifetime of the molecules is extended, although the reason for this is unclear.

The magnitude of the LGS has a strong dependence on zenith angle and Na column density, and Figure 2 shows the prediction of how they affect the beacon magnitude.

\subsection{Operational Issues}

\subsubsection{Software}

The control software has been implemented on a set of industrial VMEbus computers and UNIX workstations and is significant for reliability, usability and maintainability of the whole system. It uses EPICS for distributed realtime interprocess communication.

\subsubsection{Focus and alignment}

Two important issues which must be considered throughout any observation are the focus and alignment of the beam as these both depend on zenith angle due to the change in distance to the sodium layer, the variable alignment being a result of the offset between the axis of the launch beam and that of the telescope. A further consequence of the varying zenith angle is that the adaptive optics must be re-calibrated at intervals in order to suppress the focus term that would soon dominate.

Currently focus and alignment are both achieved manually using the telescope's TV finder. The alignment is then fine-tuned on the wavefront sensor so that the spots seen through the lenslet array on the Shack-Hartmann sensor are on average centred in the regions in which the AO algorithms operate. Once centred, the control software is able to maintain the correct orientation of the mirrors independent of the telescope's motion (a far from trivial task) by use of 3 additional pilot beams and 2 control loops for beam centring and pointing in the upper and lower sections of the beam relay.

As mentioned previously, a small intense spot is required for the centroiding algorithm to work optimally. Our typical spot size of $3^{\prime \prime}$ is near the maximum size on which it is possible to usefully correct, and attempts are being made to improve on this situation. 


\subsubsection{ALIENS}

A high power laser beam can pose a significant hazard to planes and satellites which cross its path and it is essential to include certain safety measures while the laser is operating. ALIENS (Aircraft Light Imaging Emergency Notifcation System) is a CCD camera with a $20^{\circ}$ field of view, operating in the visual waveband. It is mounted behind the secondary mirror of the main telescope to insure that it always looks in the direction that the laser is pointing. It compares consecutive frames taken with an integration time of $0.5 \mathrm{sec}$ to identify moving objects as changes that exceed a specified threshold, and on a positive detection, a shutter closes off the laser beam. Stars are automatically masked out as scintillation can trigger a false alarm.

Following significant modifications to the software, ALIENS is now in operation. Obtaining statistics on its effectiveness and reliability is slow simply because very few planes fly over Calar Alto; but it has responded to satellites and the false detection rate is typically only once every 6 hours. Difficulties still arise from high speed clouds, and from scattered light when the dome is moving.

An issue which has not been addressed concerns the possibility that the Rayleigh back-scatter may lie in the field being observed by other telescopes at the site, perhaps seriously compromising their observations.

\section{PLANS FOR THE NEAR FUTURE}

Once we are able to minimise the size of the beacon, polarisation will become an important issue. We are therefore installing an instrument to measure the polarisation at the launch telescope, together with a control loop fed back to the $\lambda / 4$-plate on the optical bench. We will also determine whether the polarisation changes with telescope position as a result of the different reflection angles.

Optimal focussing of the laser is extremely important, and using the TV guider to achieve this is clearly unsatisfactory. We propose to use the wavefront sensor, which is the most sensitive camera available to us, for this purpose by creating an optical path in the adaptive optics bench which bypasses the lenslet array so that a single spot is seen on the Shack-Hartmann sensor.

We have discussed the problems associated with using mirrors to direct the laser to the telescope. As an alternative we are experimenting with using a fibre for the same purpose, resulting both in a smaller power loss than we are currently experiencing from dirty mirrors, and also avoiding the complexity of their alignment. However, this presents a new set of difficulties. Experiments so far have been with a fused silica fibre which does not preserve the polarisation, but it is possible to buy fibres which do, or to reset the polarisation at the launch telescope. In order to retain the mono-mode quality of the beam it is necessary to use a fibre with a core diameter of only $4 \mu \mathrm{m}$, but this has knock-on effects such as increasing the Brillouin backscatter to as much as $50 \%$, and we have measured a backscatter of $1.7 \mathrm{~W}$ with an input power of $3.5 \mathrm{~W}$. It arises from the build-up of standing acoustic waves when high powers are forced into a narrow fibre, but can be suppressed by phase modulating the input beam at a frequency of about $100 \mathrm{MHz}$.

In a similar vein we are proposing to install a Raman-fibre laser during 1998/1999. In order to be competitive with our current power, the intended output will be at least $10 \mathrm{~W}$. The difference is due to the broader line-width $(2 \mathrm{GHz})$ of the fibre laser, so that most of the power is at wavelengths with a much smaller sodium absorption cross-section (the doppler broadened $\mathrm{Na}$ profile is about $2 \mathrm{GHz}$ ).

\section{EXPERIMENTS AT CALAR ALTO}

\subsection{Tip-Tilt from a Laser Beacon}

The tip and tilt modes of a wavefront distortion are responsible for the bulk of the image degradation. Although this means that image resolution can be enhanced significantly simply by use of a fast guider, it also has implications for laser guide stars because the up-link and down-link tip-tilt components are effectively the same. Thus a laser beacon cannot easily be used to measure tip-tilt modes, with the result that sky coverage is limited by the availability of stars for fast guiding. There are two alternative proposals to circumvent this difficulty. One involves the polychromatic LGS concept 5 in which the $4 \mathrm{P}_{3 / 2}$ level of $\mathrm{Na}$ is excited, and the ensuing radiative decay results in a line spectrum from $0.33 \mathrm{~nm}$ to $2.207 \mu \mathrm{m}$. The wavelength dependence of the refactive index of air allows the tip-tilt component to be determined. The other method is to use auxiliary telescopes from which the up-link and down-link motions of the laser and beacon are uncorrelated. The uncorrelated tip-tilt motions of the beacon and a natural guide star 

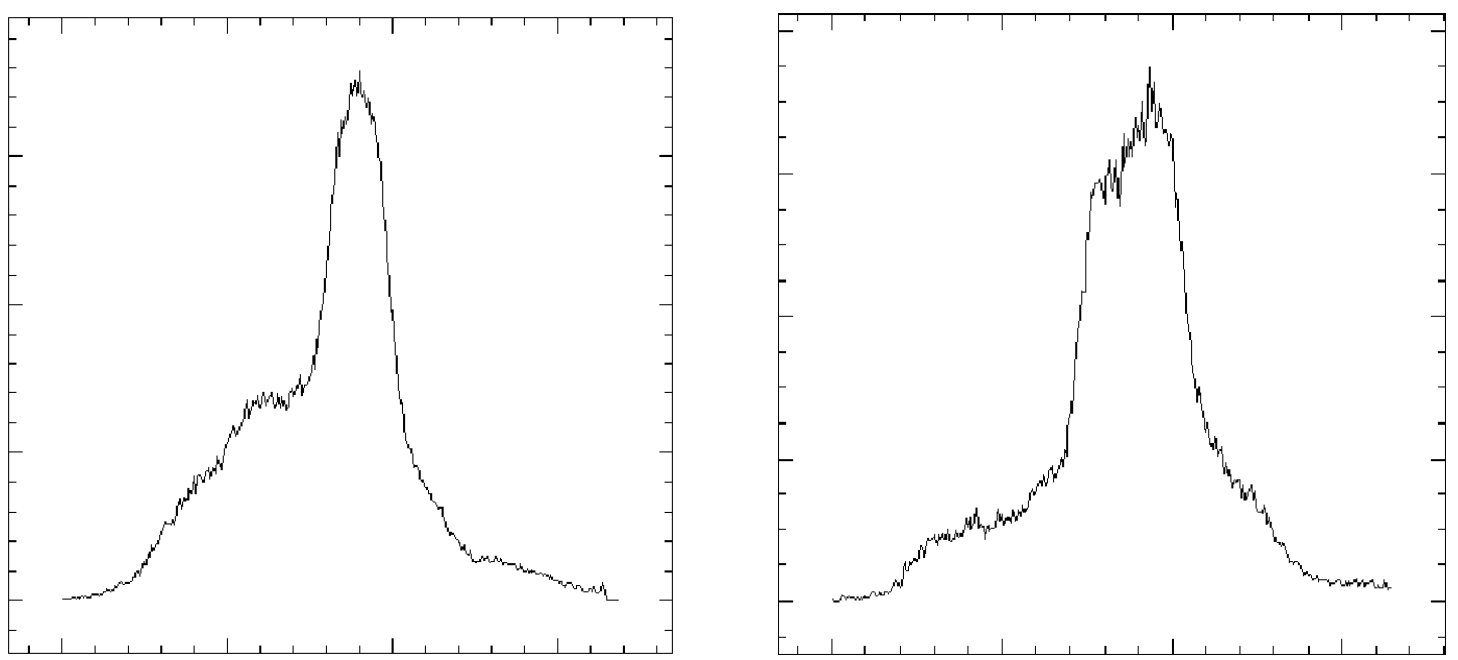

Figure 3. Profiles of the ALFA LGS plumes as seen from the $1.2-\mathrm{m}$ telescope about 1 hour apart, effectively slices through the Na layer. The horizontal axis is distance along the plume, and the vertical axis is intensity. Changes in the Na layer profile are clearly seen. The figure has kindly been provided by C. O'Sullivan and M. Redfern (University College, Galway).

are due to the up-link tip-tilt of the laser alone. The advantage is that there is a much greater chance of finding a natural star in the same isoplanatic patch as the laser, since it now appears highly elongated.

Calar Alto has two telescopes which can be used to test the latter method. In collaboration with the TMR LGS network furing 1998 we will attempt the first detection of absolute tip-tilt from a LGS system.

\subsection{Site Characteristics}

It is very important to have some knowledge of the atmospheric characterisitcs of the site if successful and optimal adaptive optics correction is to be achieved. To this end, we propose to carry out a number of experiments in collaboration with the TMR LGS network during the summer of 1998.

\subsubsection{Sodium layer profile}

At the Calar Alto site the 1.2-m and 2.2-m telesopes can be used to observe the sodium plume of the ALFA laser, which appears from them as a strip about 100" long. Previous observations during the autumn of 1997 have shown that over the time-scale of 1 hour the profile of the Na layer can change significantly, as shown in Figure 3 It is crucial to know the time-scale on which these structures appear and disappear, and the scale of the differences. One consequence is that a varying $\mathrm{Na}$ column density will affect the brightness of the laser beacon; another is that it may be necessary to refocus the launch beam, and also the wavefront sensor, on short timescales.

\subsubsection{Atmospheric turbulence profile}

The effectiveness of any given AO configuration (sampling frequency, number of modes corrected, etc.) depends on the prevailing atmospheric conditions at the time. A more practical approach is to measure the average turbulence and velocity profiles at a particular site and time of year to gain an understanding of the isoplanicity and timescale for temporal variation. The SCIDAR (SCIntillation Detection and Ranging) technique involves measuring the spatial correlation of the scintillation patterns due to a binary star in the telescope pupil plane in order to recover the refractive index structure constant $\mathrm{C}_{n}^{2}(\mathrm{~h})$ as a function of height. The method also allows the wind speed and direction of the dominant layers to be established. Some data from one night in 1997 shown in Figure 4 suggest $r_{0}=18 \mathrm{~cm}$ in the optical, an unexpectedly favourable result given the median seeing; more measurements are needed to determine the typical characteristics.

\footnotetext{
*the Network for Laser Guide Stars on 8-m class Telescopes operates under the auspices of the Training and Mobility of Researchers programme of the European Union
} 



Figure 4. SCIDAR results from Calar Alto at 3 times during a single night. The profile of the refractive index structure function $\mathrm{C}_{n}^{2}(\mathrm{~h})$ is shown, together with wind speed and direction for the dominant layers. The coherence length $r_{0}$, isopanatic angle $\theta_{0}$, and LGS focal anisoplanatic measure $d_{0}$, are calculated for $\lambda=500 \mathrm{~nm}$. The figure has kindly been provided by V. Klückers and C. Dainty (Imperial College, London)

\section{RESULTS}

The performance of the adaptive optics is integral to that of the laser beacon, and during February 1998 the it was tested extensively using a variety of subapertures, sampling frequencies, modes, and improved centroiding algorithms. Two particular examples are the achievement of an H-band FWHM of $0.24^{\prime \prime}$ on the $\mathrm{m}_{\mathrm{V}}=9.7$ star T Tau, operating at $200 \mathrm{~Hz}$ correcting 24 modes from 18 subapertures; and the ability to close the loop on the $\mathrm{m}_{\mathrm{V}}=12.3$ star GM Aurigae while correcting 7 modes from 6 subapertures at $60 \mathrm{~Hz}$.

These results indicate that the $\mathrm{AO}$ is at a stage where routine correction using a laser guide star should be possible. The detection in March 1998 of the laser on the wavefront sensor through 18 subapertures at a frame rate of $100 \mathrm{~Hz}$, as shown in Figure 5, is another indication of the progress being made. In this case we did not attempt to lock on to it due to focussing problems, but the count rate is close to that at which this should be feasible.

\subsection{The Binary System BD $+31^{\circ} 643$}

A promising result from December 1997 is the first image improvement attained by correcting on the laser guide star. A K-band image of the $0.6^{\prime \prime}$ binary $\mathrm{BD}+31^{\circ} 643$ (both B5 stars) in $1.2^{\prime \prime}$ seeing appears as a single elongated object, but correcting 7 modes on the LGS at $60 \mathrm{~Hz}$ (plus tip-tilt closed on the object at $100 \mathrm{~Hz}$ ) revealed the two stars clearly separated and with FHWM about $0.56^{\prime \prime}$. BD $+31^{\circ} 643$ has a dusty disk, and infact represents only the second detection of a circumstellar dust disk around main sequence stars, the other being around a single A0 star ( $\beta$ Pic). At a distance of $330 \mathrm{pc}$ high spatial resolution is essential for interpreting observations and furthering our understanding of dust disks around main-sequence stars, a poorly studied phase in the formation of planetesimals. In this case it indicates that binary stars may possess a stable environment for planetesimal formation. 


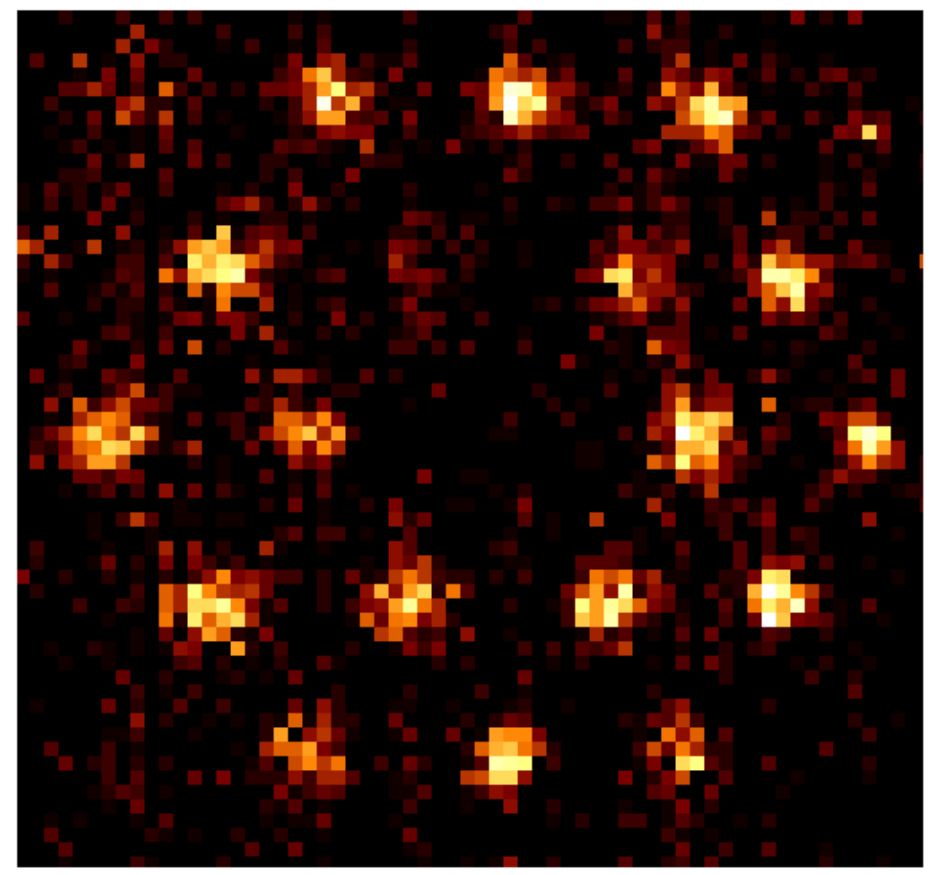

Figure 5. ALFA Laser guide star as seen on the wavefront sensor using the $5 \times 5$ lenslet array and sampling at $100 \mathrm{~Hz}$. Some problems remain with focussing and we did not attempt to lock on to it.

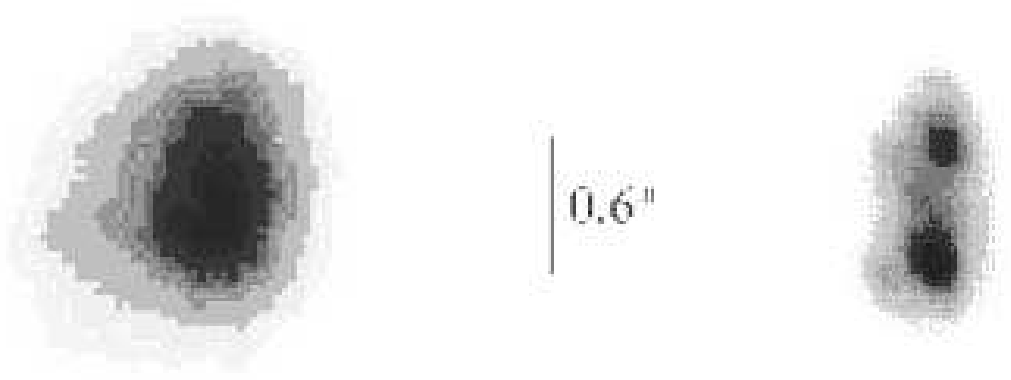

Figure 6. K-band images of $\mathrm{BD}+31^{\circ} 643$. Left is open-loop in seeing of $1.2 \mathrm{arcsec}$, and right is with the loop closed on the laser guide star to achieve a resolution of $0.56 \operatorname{arcsec}$ (see text for details). 


\subsection{Galaxies in the Abell 1367 and 262 Clusters}

Also observed in December 1997 and February 1998 were 25 galaxies in the Abell 1367 and Abell 262 clusters, including UGC 1347 and UGC 1344 (in preparation). We used a close $\left(\sim 30^{\prime \prime}\right)$ natural guide star, again correcting 7 modes at a sampling rate of $60 \mathrm{~Hz}$, achieving a disturbance rejection bandwidth of about $5 \mathrm{~Hz}$. Comparison of the closed-loop data with open-loop data taken immediately afterwards showed that the resolution had been improved from $1.2^{\prime \prime}$ to $0.9^{\prime \prime}$. For the latter we corrected on a nearby star through the same number of subapertures at a higher frequency (giving a slightly higher rejection bandwidth) and increasing the resolution in this case from $0.9^{\prime \prime}$ to $0.6^{\prime \prime}$. A second star in the same field as UGC 1347 and at approximately the same separation from the reference star could be used as a check, showing that all the sources were well within the isoplanatic patch and that the reference stars represented an accurate portrayal of the point spread function to clean the images.

The detailed study of these two galaxies showed that there is evidence for nuclear star formation, and that part of the nuclear K-band luminosity can be explained by the presence of a young stellar population. The larger sample of galaxies indicates a correlation between the size of the galaxy bulge in the K-band, and the distance from the centre of the cluster. This is explained in terms of enhanced star formation in gas-rich spirals triggered by galaxy interactions as they cross the central regions of the cluster. The additional compact component of flux from this activity then influences the apparent size of the bulge. The typical bulge size is $\lesssim 1^{\prime \prime}(\lesssim 300 \mathrm{pc})$, and high spatial resolution is essential to measure this accurately as provided by adaptive optics with use of a LGS.

\section{ACKNOWLEDGMENTS}

The MPIA/MPE team thanks the Calar Alto staff for their help and hospitality, and N. Wilnhammer for technical support. RID acknowledges the support of the TMR (Training and Mobility of Researchers) programme as part of the European Network for Laser Guide Stars on 8-m Class Telescopes. We thank Mike Redfern and Creidhe O'Sullivan (University College, Galway), and Vince Klückers and Chris Dainty (Imperial College, London) who provided the figures for Section 4.2 .

\section{REFERENCES}

1. A. Glindemann, D. Hamilton, S. Hippler, R.-R. Rohloff, and K. Wagner, "ALFA - The laser guide star adaptive optics system for the Calar Alto 3.5-m telescope," in Laser Technology for Laser Guide Star Adaptive Optics Astronomy, N. Hubin and H.Friedmann, eds., pp. 120-125, ESO, 1997.

2. S. Hippler, A. Glindemann, M. Kasper, P. Kalas, R.-R. Rohloff, K. Wagner, D. Looze, and W. Hackenberg, "ALFA : The MPIA/MPE adaptive optics with lasers for astronomy project," in Adaptive Optical System Technologies, Proc. SPIE 3353, 1998. Paper 05.

3. A. Quirrenbach, W. Hackenberg, H.-C. Holstenberg, and N. Wilnhammer, "The ALFA dye laser system," in Laser Technology for Laser Guide Star Adaptive Optics Astronomy, N. Hubin and H.Friedmann, eds., pp. 126-131, ESO, 1997.

4. A. Quirrenbach, W. Hackenberg, H.-C. Holstenberg, and N. Wilnhammer, "The sodium laser guide star system of ALFA," in Adaptive Optics and Applications, Proc. SPIE 3126, pp. 35-43, 1997.

5. R. Foy, A. Migus, F. Biraben, G. Grynberg, P. McCullough, and M. Tallon, "The poylchromatic artificial sodium star: a new concept for correcting the atmospheric tilt," A\&AS 111, p. 569, 1995.

6. R. Ragazzoni, S. Esposito, and E. Marchetti, "Auxiliary telescopes for the absolute tip-tilt determination of a laser guide star," MNRAS 276, p. P76, 1995.

7. P. Kalas and D. Jewitt, "A candidate dust disk surrounding the binary stellar system BD $+31^{\circ} 643$," Nature 386, pp. 52-54, 1997. 